\title{
Activités
}

11-1| Avril 2014

Varia

\section{La performance arbitrale : de son étude à son développement}

Refereeing performance: From study to development

Géraldine Rix-Lièvre, Simon Boyer, Fabien Coutarel et Pascal Lièvre

\section{OpenEdition}

1 Journals

Édition électronique

URL : http://journals.openedition.org/activites/425

DOI : 10.4000/activites.425

ISSN : 1765-2723

Éditeur

ARPACT - Association Recherches et Pratiques sur les ACTivités

Référence électronique

Géraldine Rix-Lièvre, Simon Boyer, Fabien Coutarel et Pascal Lièvre, «La performance arbitrale : de son étude à son développement ». Activités [En ligne], 11-1 | Avril 2014, mis en ligne le 15 avril 2014, consulté le 30 avril 2019. URL : http://journals.openedition.org/activites/425 ; DOI : 10.4000/ activites. 425

\section{(c) $(1) \Theta \Theta$}

Activités est mis à disposition selon les termes de la licence Creative Commons Attribution - Pas d'Utilisation Commerciale - Pas de Modification 4.0 International. 


\title{
La performance arbitrale : de son étude à son développement
}

\section{Géraldine Rix-Lièvre}

Laboratoire ACTé (EA 4281), Clermont Université, Université Blaise Pascal, UFR STAPS, BP 10448, F-63000 Clermont-Ferrand-Geraldine.Rix@univ-bpclermont.fr

\section{Simon Boyer}

Laboratoire ACTé (EA 4281), Clermont Université, Université Blaise Pascal \& Groupe La Poste BP 10448, F-63000 Clermont-Ferrand - Simon.Boyer@univ-bpclermont.fr

\section{Fabien Coutarel}

Laboratoire ACTé (EA 4281), Clermont Université, Université Blaise Pascal, UFR STAPS, BP 10448, F-63000 Clermont-Ferrand - Fabien.Coutarel@univ-bpclermont.fr

\section{Pascal Lièvre}

Laboratoire CRCGM (EA 3849), Clermont Université, Université d'Auvergne, BP 10448, F-63000 Clermont-Ferrand - PascalLievre@orange.fr

\begin{abstract}
Refereeing performance: From study to development. . The purpose of this article is to analyze refereeing performance in sports. Two studies were carried out: the first focused on the referee's effective activity and experience; the second examined the supervisor's activity, as he assesses the referee's performance. We show that: (1) The referee's actual work exceeds the tasks he is usually assigned - that of enforcing the rules; (2) refereeing involves the development of multiple skills so as to show players what is allowed and ensure their compliance; (3) refereeing performance is co-constructed. The study on supervisors' assessment activity complements these results, by highlighting the tensions which comprise the construction of refereeing performance: (1) tension between a performance built on the accuracy of the referee's decisions in relation to separate situations within the game, and consideration of refereeing dynamics throughout the match; (2) tension between a performance given in accordance with objective elements and an assignment of value which depends on a community's interests; (3) tension between the a priori prescribed criteria and certain elements of contextualized assessment. This research also demonstrates the complexity and the collective and systemic nature of refereeing performance. In so doing, it provides new opportunities for the development of refereeing performance, based on work debates.
\end{abstract}

KEY WORDS

rugby referee, experience, skills, performance, evaluation, work debates.

\section{Introduction}

L'arbitrage sportif est une activité en pleine mutation. Depuis toujours, alors que l'arbitre est indispensable au déroulement d'une rencontre sportive, il est décrié : "Que les joueurs se battent, c'est la faute à l'arbitre : il n'est pas assez sévère, il ne siffle pas assez! Siffle-t-il ? 
Il siffle trop, il gâche le plaisir des joueurs et des spectateurs, le match est de mauvaise qualité, c'est la faute à l'arbitre!»(Austry, 2000). Aujourd'hui la demande sportive et sociale est de plus en plus exigeante : l'arbitre de haut-niveau doit être irréprochable, plus professionnel que les joueurs professionnels... sans nécessairement avoir ce statut. L'évolution rapide du système sportif, qui est devenu un vrai secteur professionnel concurrentiel, suppose une évolution de l'arbitrage. La question du statut de l'arbitre devient prégnante, mais à l'heure où l'arbitrage pourrait devenir professionnel ou semiprofessionnel, les modalités de construction du métier ne sont que peu interrogées. Pour l'instant, la performance est très individualisée : seule la prestation de l'arbitre est sous les feux de la rampe et sous le regard des chercheurs qui s'attachent surtout à déterminer l'exactitude des décisions par rapport au règlement (Mascarenhas, Button, O'Hare, \& Dicks, 2009). La performance arbitrale doit selon nous être re-questionnée d'une part pour prendre en charge sa complexité, d'autre part pour que l'étude de cette dernière participe à son développement. Il s'agit, comme le propose la clinique de l'activité (Clot, 1999; Clot, \& Faïta, 2000), de prendre en charge les dimensions collective et systémique de la performance pour favoriser et accompagner la construction collective du métier.

Ainsi, nous nous proposons, dans le cadre de cet article, d'étudier la performance arbitrale et d'envisager une voie de construction du métier. Ce faisant, nous nous intéressons à l'arbitrage sportif de haut-niveau en tant que tel, mais aussi comme un cas exemplaire. Ce cas est exemplaire à au moins double titre. D'une part, l'exposition extrême de l'arbitre rend accessible des processus de construction et de remise en cause de la performance, qui existent pour d'autres activités professionnelles, mais dont le caractère plus confidentiel contribue à les rendre moins facilement saisissables. D'autre part, l'évolution récente du sport vers la professionnalisation structure un environnement nouveau autour de l'arbitre : un métier est en train de se construire. Nous présentons dans un premier temps une étude de l'activité de l'arbitre en match qui nous a permis d'envisager l'arbitrage au-delà de la tâche d'application des règles qui lui est assignée. Puis, nous proposons d'éclairer, au-delà de la prestation de l'arbitre, certains déterminants de la performance arbitrale en montrant les modalités selon lesquelles l'établissent les superviseurs qui ont pour fonction d'évaluer la prestation de l'arbitre sur le terrain lors d'un match particulier. Les résultats de ces différentes études nous conduisent enfin à discuter une voie de développement de la performance arbitrale allant de concert avec une perspective de construction du métier.

\section{1.- L'activité de l'arbitre de haut-niveau aujourd'hui}

\section{1.- De l'application de la règle aux actes de jugement de l'arbitre}

Relativement peu de travaux de recherche s'attachent à l'arbitrage. La plupart d'entre eux s'inscrivent dans un formalisme juridique. Celui-ci peut être décliné en trois points : (1) la faute est factuelle, (2) la règle détermine les jugements, (3) la tâche de l'arbitre est de bien connaître la règle et de bien voir les faits. Certains travaux concernent le potentiel physique de l'arbitre, son impact sur sa performance et les possibilités d'entraînement (Kay, \& Gill, 2003 ; Weston, Castagna, Impellizzeri, Rampinini, \& Abt, 2007). D'autres se centrent sur les processus de décision de l'arbitre et ses biais : biais perceptifs, liés à la catégorisation, à la mémorisation ou à l'intégration d'informations (Dosseville, \& Garncarzyk, 2007 ; Plessner, \& Haar, 2006). De manière expérimentale, les éléments influençant la discrimination visuelle ou l'évaluation de la loyauté de l'action sont appréhendés. D'autres résultats soulignent surtout que les décisions prises par l'arbitre lors du match sont différentes de celles prises au cours des protocoles expérimentaux (Gilbert \& Trudel, 2000 ; Rainey, Larsen, \& Williard, 1987 ; Trudel, Dionne, \& Bernard, 2000). Enfin, l'examen des comportements de l'arbitre en match montre que si l'arbitre, pour $40 \%$ du temps, prend des décisions, il consacre plus de $50 \%$ du temps à interagir avec les joueurs (Trudel, Cote, \& Sylvestre, 1996). Par conséquent, en match, l'activité de jugement de l'arbitre ne peut être 
restreinte à des prises de décisions relatives à la conformité de la configuration du jeu par rapport aux règles.

L'étude des situations effectives d'arbitrage (« réelles » dirait-on en ergonomie) s'avère indispensable pour comprendre cette activité d'une part, et accompagner son évolution d'autre part. Il s'agit donc de se détacher de tout formalisme juridique pour appréhender ce que fait l'arbitre au moment même où il arbitre (Rains, 1984; Rix, 2005 ; Russell, 1997, 1999). Différents écrits sur l'acte de juger des magistrats (Perelman, 1990 ; Ricœur, 1995) contribuent à cette démarche en définissant tout jugement par son caractère actif et non déterminé : ils le distinguent ainsi du résultat - déterminable a priori - d'un processus mécanique inscrit dans des normes écrites (Oakeshott, 1995). L'acte de juger est alors identifié relativement à ce qu'il produit : il montre et impose ce qui est possible en référence tant au contenu d'idéaux qu'à ce qui est légal, et ce faisant, il établit et qualifie les évènements (Ricœur, 1995). L'activité de jugement de l'arbitre est donc envisagée comme des actes qui, dans leurs manifestations langagières et/ou gestuelles, montrent ce qui est possible et, en l'imposant, lèvent l'incertitude des événements.

\section{2.- Investir l'expérience de l'arbitre pour comprendre son activité en match}

La possibilité d'investir l'expérience de l'arbitre repose avant tout sur une observation participante. Cette observation participante consiste en une intégration progressive au sein de la communauté des arbitres de rugby en participant à des stages, à des réunions, à des rencontres informelles ou encore en assistant à des matches et des tournois. Cette intégration permet une première compréhension, intuitive, de l'activité de l'arbitre. Mais elle correspond aussi à la construction progressive de la place du chercheur dans le système qu'il investit (Favret-Saada, 2009). Au cours de ce travail de recherche, il était important de construire une posture permettant à l'arbitre de livrer au chercheur ce qu'il vit en match sans crainte d'être jugé ou une fois encore critiqué. Contrairement à d'autres travaux, notre posture est clairement engagée: il s'agit de montrer comment l'arbitre, malgré un environnement dynamique et incertain, des problèmes mal structurés, des objectifs multiples et concurrentiels, des conditions changeantes, la pression temporelle, l'importance des enjeux, la multiplicité des acteurs et les contraintes organisationnelles, est en mesure de prendre des décisions en match (Mascarenhas, Collins, Mortimer, \& Morris, 2005 ; Rix-Lièvre, Boyer, \& Récopé, 2011). Pour mener ce projet, notre engagement sur le terrain correspondait à la posture d'une personne naïve qui veut apprendre des acteurs et relevait d'une attitude compréhensive. C'est à cette condition qu'une confiance mutuelle a pu être construite entre le chercheur et chaque arbitre ayant participé à l'étude.

Pour comprendre l'activité de l'arbitre en match à partir de son expérience, plusieurs investigations plus spécifiques ont été menées lors de sept matches de rugby au plus hautniveau amateur (Fédérale 1) et de quatre matches au plus haut-niveau professionnel (Top 14). Pour chaque investigation, le match faisant l'objet de l'étude a été défini en collaboration avec la Fédération Française de Rugby. Dès lors tous les acteurs du match étaient contactés par téléphone afin de leur présenter l'objectif du travail et le protocole mis en place.

Le jour du match, arrivé au stade avec l'arbitre, le chercheur le suit dans ses activités et met en place différents dispositifs permettant de réaliser simultanément deux enregistrements vidéo : (1) un enregistrement du match en plan large à partir des tribunes qui constitue une trace des comportements de l'arbitre et des joueurs, (2) un enregistrement audio-vidéo d'une perspective proche de celle de l'arbitre en situation, perspective dite subjective située (Rix \& Biache, 2004). Cette perspective est construite en équipant l'arbitre d'une caméra (Ø $8 \mathrm{~mm})$ fixée au niveau de sa tempe et d'un système d'enregistrement embarqué ( 3 boîtiers de respectivement $8 \times 5 \times 2 \mathrm{~cm}, 9 \times 5 \times 1,5 \mathrm{~cm}, 7,5 \times 6,5 \times 2 \mathrm{~cm}$, pesant au total $350 \mathrm{~g}$ ) placé dans une ceinture identique à celle utilisée pour porter les appareils de transmission audio. Ainsi, deux traces de l'activité de l'arbitre pendant la rencontre sont élaborées. 
Après chaque match - soit juste après la rencontre, soit dans la semaine suivante - , l'arbitre est sollicité pour un entretien en re situ subjectif (Rix, 2005 ; Rix, \& Biache, 2004 ; RixLièvre, 2010). Cet entretien inspiré de l'entretien d'explicitation (Vermersch, 1994) et de l'entretien d'auto-confrontation (Theureau, 1992), vise à revenir avec l'arbitre sur son vécu tout au long du match. Il s'agit d'utiliser la perspective subjective située pour re-placer l'acteur, lors de l'entretien, dans une perspective proche de son point de vue en situation. Cette nouvelle trace vise à faciliter tant l'effort de ré-flexion de l'acteur que la conduite - par le chercheur - de l'entretien vers une explicitation de l'action. Cette situation d'entretien "permet à l'acteur d'accompagner le chercheur dans ce qui, au moment de la pratique, fait sens pour lui» (Rix, 2005, p.275). Ce dispositif méthodologique utilisé pour étudier différentes pratiques - pratiques d'expéditeurs polaires à ski, de prestataires de loisirs sportifs, d'entrepreneurs, de joueurs de rugby ou encore d'ouvriers - est un outil de constitution de l'expérience permettant de mettre à jour des dimensions cognitives de l'activité (Rix-Lièvre, 2010).

Les verbalisations issues de l'entretien en re situ subjectif sont ensuite traitées de concert avec des matériaux d'observation afin de formaliser, en partant de l'expérience dévoilée, le déroulement de la pratique de l'acteur. Le corpus est ainsi organisé sous forme de tableaux dans lesquels sont mis en parallèle, le déroulement temporel de l'acte, des matériaux d'observation et la manière dont l'arbitre en rend compte au cours de l'entretien en re situ subjectif. Cette mise en parallèle permet une formalisation synthétique de la manière dont, dans la singularité du moment, l'arbitre en vient à montrer et imposer aux joueurs ce qui est possible en prenant en compte tant le vécu de l'acteur que ce qu'il a effectivement réalisé. C'est à partir de ces formalisations que différentes catégories sont construites. Elles le sont de manière itérative en comparant et distinguant ce que l'arbitre impose et les modalités de construction de ce qui est imposé.

\section{3.- Une conception alternative de l'arbitrage : de la tâche à l'activité}

\subsection{1.- Différents types d'acte de jugement pour décrire l'activité de l'arbitre}

Nos travaux ont permis de circonscrire trois types d'acte de jugement (Rix, 2005): un jugement-en-acte, un jugement de fait et un jugement délibéré. Ceux-ci se distinguent relativement à ce qui est imposé (la nature et la légitimité de ce que l'arbitre impose, les modalités selon lesquelles il lève l'incertitude de la situation) et au mode de construction de ce qui est imposé (l'état du jeu lorsque la construction s'effectue, la manière dont l'arbitre appréhende spontanément les actions des joueurs, la temporalité de la construction).

Le jugement-en-acte est un acte de jugement qui s'élabore progressivement dans le rapport dynamique de l'arbitre à ce que font les joueurs. Ainsi, ce que montre et impose l'arbitre, de l'ordre des manières d'agir des joueurs, se construit au fil de son engagement au cœur de l'opposition et n'existe qu'à l'instant où il le rend manifeste dans un geste, une parole et/ou un coup de sifflet. Si le jugement-en-acte est souvent rapporté en rugby à l'arbitrage de l'avantage où l'arbitre considère la dynamique de l'activité des joueurs, il est aussi repérable dans bien d'autres séquences, par exemple dans le moment suivant.

Suite à une touche, les blancs ont récupéré le ballon et, au contact des adversaires, formé un maul qui progresse le long de la ligne de touche. L'arbitre qui était placé au fond de la touche, revient près du maul et avance avec lui en restant grand côté, c'est-à-dire du côté du terrain par rapport au maul où la ligne de touche est la plus éloignée. Le maul resté stationnaire une fois, est reparti, puis s'est à nouveau arrêté. L'arbitre indique aux joueurs blancs qu'ils doivent sortir la balle, s'ils la sortent, le jeu peut se poursuivre. Comme ils ne la sortent pas, l'arbitre siffle, les blancs perdent la balle. Il les a prévenu, il leur a dit ce qu'il fallait faire, ils ne l'ont pas fait, il donne la balle à l'adversaire. Il accompagne son coup de sifflet d'une explication : il leur demande de faire quelque chose, ils ne le font pas, le maul est bloqué et redémarre à deux reprises, il attribue donc une mêlée aux oranges. 
Dans cet exemple, l'existence de la faute dépend de la manière dont les joueurs prennent en compte ce qu'impose l'arbitre puisque s'ils sortent le ballon, le jeu se poursuit sans faute, s'ils ne le sortent pas, l'arbitre siffle. Le fait qu'il y ait ou non faute ne pré-existe pas à cette dynamique. Ainsi, le jugement-en-acte est un moment judicatoire : ce qui est pointé et imposé par l'arbitre, relativement aux manières d'agir des joueurs, d'interagir entre eux et/ou avec l'arbitre, devient nécessaire pour lui dans un mouvement. C'est au fil de son interaction avec le/les joueur(s) que l'arbitre en vient à signaler une faute : siffler devient nécessaire. Cette nécessité qui reste phénoménale, est légitimée par l'arbitre à travers la convocation immédiate d'une règle dans ce qu'elle édicte, ici celle relative à la progression d'un maul.

Le jugement de fait correspond à un acte de jugement où l'arbitre constate et impose un fait rapporté à une règle précise. L'arbitre appréhende l'activité des joueurs comme un comportement figé, un élément précis, comme un fait établi d'emblée rapporté à une règle particulière. Cet élément s'impose à lui comme une réalité évidente : l'établissement du fait qui ne suppose ni preuve, ni justification et porte sur quelque chose d'actuel, est de l'ordre d'une évidence (Gil, 1993). L'exemple suivant illustre ce que nous considérons comme un jugement de fait.

Mêlée au centre du terrain pour les verts, le demi-de-mêlée sort le ballon de la mêlée et le passe sur sa droite, l'arbitre placé côté introduction au départ (c'est-à-dire à gauche) suit la direction de la trajectoire du ballon en passant derrière les joueurs verts encore en mêlée. Le joueur vert ayant réceptionné le ballon fait lui aussi une passe à un coéquipier sur sa droite qui lui s'engage dans la défense adverse. Un blanc tente de le plaquer, le porteur du ballon reste debout, un autre blanc vient le ceinturer en même temps qu'il passe la balle à un autre vert venu derrière au soutien. Le porteur du ballon revenant à l'intérieur rentre dans un partenaire situé en avant de lui par rapport à la ligne de but adverse. L'arbitre siffle tout en signalant le contact, même s'il est navré de stopper l'action de jeu. Il se justifie auprès des joueurs : y a contact.

L'arbitre constate un fait dans l'instant : "y a contact ». Le contact est perçu comme une réalité discernable et évidente. C'est un fait qui n'a d'existence que relativement tant à quelque chose d'actuel qu'à une règle. Dès lors, on pourrait penser que ce type d'acte de jugement renvoie à un certain type de faute qui serait rapportable à un fait constatable. Ainsi, certaines dérogations au règlement pourraient en fonction de leurs formulations être établies de manière factuelle, comme un en-avant, une touche, un engagement qui ne fait pas 10 mètres... Ces fautes pourraient par définition se rapporter à un constat d'évidence, comme d'ailleurs l'essai, le but et l'arrêt de volée qui relèvent aussi, dans les règles, d'un fait constatable selon une logique binaire. Mais le jugement de fait ne relève pas d'une logique binaire supposant d'envisager s'il y a ou non faute, c'est-à-dire de mettre en rapport une configuration de jeu donnée à une règle préexistante. Il repose sur une évidence perceptive dans laquelle le fait et la règle sont simultanément manifestes dans l'instant. C'est cette évidence que l'arbitre montre et impose aux joueurs. D'ailleurs, dans l'exemple présenté, l'arbitre juge un fait, mais ce n'est pas une faute dont la formulation règlementaire permet de poser comme observable et déterminée selon une logique binaire. En effet, le contact est rapportable à la règle suivante : "Lorsqu'un joueur hors-jeu ne peut pas éviter d'être touché par le ballon ou par un coéquipier qui le porte, il est hors-jeu accidentellement. Si l'équipe du joueur n'en tire pas d'avantage, le jeu doit continuer. Si elle en tire un avantage, une mêlée doit être ordonnée, l'équipe adverse bénéficiant de l'introduction" (Commission Centrale des Arbitres, 2002, p. 63). Ainsi, la formulation de la règle pose des éléments factuels, mais ils ne sont pas suffisants pour établir la faute; pour ce faire, la règle stipule que l'avantage qu'en tire l'équipe fautive doit être considéré. Par conséquent, le jugement de fait ne se rapporte pas à un type de faute qu'il serait possible d'établir factuellement dans une logique binaire: ce type d'acte de jugement caractérise une manière pour l'arbitre d'appréhender l'activité des joueurs. 
Le jugement délibéré se différencie des deux précédents dans la mesure où il se développe non plus en cours de jeu, mais une fois que celui-ci est suspendu. Il n'est repérable que lors de situations problématiques pour l'arbitre dans lesquelles il prend un temps d'enquête, de réflexion, de discussion avec ses assesseurs. Alors que le jeu est arrêté, il examine avec plus d'informations, d'avis et/ou de recul ce qui s'est passé afin d'en produire in fine un scénario plausible, cohérent, acceptable qui détermine la façon dont l'opposition se poursuivra. La sollicitation de l'arbitre vidéo dans les championnats professionnels est un exemple archétypal. Lors d'une action proche de la ligne de but ou dans l'en-but, l'arbitre arrête le jeu et sollicite, après ou non avoir consulté ses assistants, l'arbitre vidéo en lui posant une question en fonction de la manière dont il a appréhendé l'activité des joueurs au cours de la séquence en question. Si la sollicitation de l'arbitre vidéo relève toujours d'un jugement délibéré de l'arbitre de champ, d'autres jugements délibérés sont repérables; c'est le cas dans l'exemple suivant.

Mêlée, le ballon est talonné par les oranges, le demi-de-mêlée sort le ballon, puis s'engage petit côté. L'arbitre part avec l'action : il suit la progression des oranges porteurs du ballon. Cependant, il continue de se préoccuper de la mêlée. Tout en courant dans le sens de l'action de jeu, il se retourne pour regarder l'emplacement de la mêlée : les joueurs sont toujours groupés, ils ne bougent pas, ils ne viennent pas au jeu. Le porteur du ballon poursuit son action. L'arbitre qui le suit toujours se retourne une nouvelle fois, il voit alors que le conflit ne se calme pas, il siffle pour arrêter le jeu et revient en courant à l'emplacement de la mêlée.

Il s'attache à calmer les joueurs qui se battent et surtout à éviter que la bagarre prenne de l'ampleur. Puis, il sépare les deux équipes et les écarte le plus possible pour que le problème ne recommence pas. Il stipule aux capitaines d'attendre pendant qu'il va voir son juge de touche pour prendre des informations.

Il rejoint rapidement un de ses juges de touche ; celui qui, du côté du conflit, est entré sur le terrain. Dès qu'ils sont côte-à-côte, le juge de touche précise à l'arbitre ce qu'il a vu : pendant la mêlée, le deuxième ligne orange qui relève, puis le 5 noir qui réplique par un coup de poing. L'arbitre prend une vingtaine de secondes avec ce juge de touche pour s'assurer qu'ils sont d'accord sur ce qui s'est passé et sur la sanction à imposer: les deux équipes sont fautives, mais c'est un joueur orange qui est à l'origine du problème, une pénalité sera attribuée aux noirs.

Il revient ensuite près des capitaines : "D'accord, donc c'est un deuxième ligne orange qui relève, rendu par le 5 noir! Première faute orange, premier avertissement sans sanction, après y aura sanction. D'accord, on est d'accord? ». Il termine en demandant à chacun des capitaines de tenir son équipe pour que le match se déroule le mieux possible, puis il leur signale qu'une pénalité noire sera jouée avant de les envoyer près de leurs joueurs pour les prévenir qu'ils doivent se concentrer sur le jeu.

Dans cette séquence, l'arbitre est contraint d'arrêter le jeu. Une fois le jeu arrêté, il prend le temps d'aplanir le problème, de calmer les joueurs. Le jeu est ainsi suspendu pour rétablir des conditions d'opposition acceptables, mais aussi pour laisser le temps à l'arbitre de s'informer auprès de son juge de touche sur ce qui s'est passé. En faisant le point avec son assesseur, il recueille, par son témoignage, des informations sur l'événement passé. Il est alors important de reconstruire le déroulement de cet événement pour déterminer la ou les fautes que l'arbitre va pointer et sanctionner. Ainsi, au cours même du match, l'acte de jugement de l'arbitre repose sur un examen conscient et réfléchi de ce qu'il s'est passé et de la manière dont le jeu doit reprendre. Le scénario alors construit est une manière de décrire ce qu'il s'est passé en le connotant d'emblée par rapport à la transgression ou non de certaines règles. En indiquant "d'accord, donc c'est un deuxième ligne orange qui relève, rendu par le 5 noir! », l'arbitre se centre sur une faute dans le jeu et fait reprendre le match par une pénalité sans attribuer de carton aux joueurs relativement au conflit. Les termes qu'il emploie constituent une manière de qualifier l'événement dont découlent logiquement les modalités de reprise du jeu.

La formalisation de ces différents types d'actes de jugement met à jour la complexité de 
l'activité de l'arbitre. Chaque acte de jugement repose sur des connaissances et compétences différentes ; ce qui suppose pour l'arbitre de les construire toutes.

Les jugements-en-acte reposent largement sur des connaissances implicites de ce qu'est l'activité sportive arbitrée, c'est-à-dire sur ce que, au cours de l'opposition, l'arbitre considère spontanément comme étant ou non du rugby. Ces connaissances se construisent sur le tas, au fil de son engagement dans le rugby et dans l'arbitrage. Elles fondent ce qui est souvent posé comme une saisie intuitive du jeu. Inversement, le jugement délibéré suppose une analyse réfléchie, mais malgré tout rapide, de différents éléments portés à la connaissance de l'arbitre pour construire un scénario logique.

Les différents types d'acte de jugement engagent aussi l'arbitre dans des modalités d'interaction différentes avec les joueurs. Par exemple, le jugement de fait n'a pas à être expliqué dans la mesure où le fait est évident et directement lié à une règle alors que le jugement-en-acte suppose de rapporter ce qui est imposé à une règle et de rendre ce rapport visible pour les joueurs. Ainsi, l'arbitre doit construire différentes modalités de coordination avec les acteurs de la rencontre.

\subsection{2.- Vers une nouvelle manière d'envisager l'activité d'arbitrage et la performance arbitrale}

Ces résultats contribuent aussi à la construction d'une conception alternative de l'arbitrage (Rix, 2005), où l'activité de l'arbitre dépasse largement la tâche qui lui est généralement assignée : appliquer le règlement. Ses coups de sifflet, propos et gestes ne sont plus considérés comme le résultat prévisible d'une analyse de la conformité du jeu par rapport au règlement, mais comme des manifestations d'actes de jugement qui montrent et imposent aux joueurs ce qui est possible à un moment donné. Chaque acte de jugement de l'arbitre décrit de façon particulière une situation comme un fait, une dynamique ou un problème ; cette description est performative au sens où elle fait advenir ce qu'elle décrit, de la manière dont elle le décrit. Autrement dit, la faute n'est plus relative à la réalité d'un fait, mais dépend du rapport de l'arbitre aux actions des joueurs. Comme le souligne Serres (2010, pp. 14-15), "c'est donc une erreur [...] que de dire : le but ou l'essai y était ou n'y était pas et l'arbitre l'a accepté ou refusé. Non le but y est dès lors que l'arbitre l'a ainsi décidé. [...] Un but est un événement juridique décidé, mieux, créé même par l'arbitre ». Il ne s'agit pas de considérer que l'arbitre fait le jeu ou invente des buts, mais de souligner que même si l'activité des joueurs préexiste aux actes de jugement, c'est la manière dont l'arbitre les montre qui les fait advenir en tant qu'événements du jeu - but, essai, faute... Ainsi, l'arbitre co-construit, avec les joueurs, le déroulement du jeu et les règles ne déterminent plus son activité, mais permettent, au sens de pouvoir et de possibilité, cette co-construction.

Ces résultats ont plusieurs conséquences. Cette étude de l'activité des arbitres en match en partant de leur expérience en situation met en exergue l'écart important entre ce qui est souvent considéré comme la tâche de l'arbitre - appliquer des règles - et son activité effective. Ainsi, la qualité de la prestation de l'arbitre ne peut être rapportée au simple critère formel d'application de la règle. Si l'arbitrage consiste à co-construire avec les joueurs le déroulement du jeu, il devient alors aussi impossible d'isoler la performance de l'arbitre de la prestation des joueurs. Il s'agit d'ores et déjà d'admettre que cette performance, comme dans d'autres métiers dans le domaine des services, est largement co-construite (Motté, \& Haradji, 2007 ; Valléry, \& Leduc, 2005). Enfin, ces résultats nous invitent à prendre en charge la complexité de la performance arbitrale et son inscription dans des situations singulières. 


\section{2.- L'inscription de la performance arbitrale dans un système : une étude de l'activité du superviseur}

Si les résultats précédents nous conduisent à désindividualiser en partie la performance arbitrale en admettant qu'elle est co-construite avec les acteurs de la rencontre, notamment les joueurs, il faut aussi souligner qu'une prestation n'est performance que si elle est désignée comme telle par les institutions. En effet, un arbitre n'est arbitre de haut-niveau que si le système reconnaît sa compétence à arbitrer et le désigne pour officier au sein des championnats les plus prestigieux. Ainsi, la performance arbitrale doit être rapportée tant à une production sur le terrain (ou plutôt à une co-production) qu'à la manière dont cette dernière est évaluée. Par conséquent, après avoir étudié l'activité effective de l'arbitre sur le terrain, nous nous sommes intéressés à la manière dont l'institution/le système établissait la performance arbitrale. Pour ce faire, nous avons investigué en premier lieu l'activité de la personne chargée d'évaluer la prestation de l'arbitre en match : le superviseur.

Nommé quelques jours avant la rencontre, le superviseur vient sur le lieu du match pour apprécier la performance de l'arbitre. Une fois le match terminé, il indique à l'arbitre les éléments positifs et négatifs de sa prestation. Un échange peut aussi avoir lieu dans les jours qui suivent. In fine, le superviseur rédige un rapport adressé à la fédération ; rapport auquel l'arbitre de la rencontre a directement accès lorsqu'il officie à haut-niveau. L'ensemble des rapports effectués tout au long d'une saison sportive participe au classement des arbitres les uns par rapport aux autres. Certains sont confortés dans leurs capacités à diriger des rencontres d'un niveau de championnat donné. D'autres sont testés dans des divisions supérieures. D'autres encore descendent de division, remplacés la saison suivante par ceux qui montent. Ainsi par son rapport, le superviseur assume un rôle clé dans l'établissement institutionnel d'une performance arbitrale. C'est pourquoi nous avons choisi, même si d'autres acteurs sont importants dans ce processus, d'étudier l'activité du superviseur. Nous nous sommes centrés non sur les discours des superviseurs à propos de leur propre activité et/ou de la performance arbitrale, mais sur la manière dont un superviseur évalue effectivement la prestation de l'arbitre. Ce travail vient compléter la recherche précédente à double titre :

— il doit mettre en évidence la manière dont une valeur est attribuée à l'arbitrage lors d'une rencontre particulière. Ce faisant, il inscrit complètement la performance arbitrale dans un système : elle se construit à la fois sur le terrain de jeu et en dehors de celui-ci ;

— il doit aussi contribuer à l'identification des attentes qui pèsent sur la prestation de l'arbitre sur le terrain. Ainsi, les dimensions collectives et systémiques de la performance arbitrale pourront être éclairées.

\section{1.- Investir l'activité des superviseurs}

Il faut tout d'abord préciser que cette étude, menée par la même équipe de recherche, s'inscrit dans la même posture compréhensive et engagée que la précédente et dans une continuité de collaboration avec la Fédération Française de Rugby. Ainsi, elle profite de tout le travail d'imprégnation effectué au cours de l'intégration progressive des chercheurs au sein de la communauté des arbitres de rugby. Comme pour l'étude de l'activité des arbitres en match, cette nouvelle investigation repose aussi sur une démarche d'observation participante.

Au-delà de cette imprégnation globale, la démarche mise en œuvre lors de chaque rencontre où l'activité du superviseur a été étudiée doit être précisée. Pour chaque investigation, le match faisant l'objet de l'étude a été défini en collaboration avec la Fédération Française de Rugby. Nous pouvons ainsi considérer que nous avons été confrontés aux pratiques que la fédération valorise. Quatre matches des championnats professionnels français ont été investigués : trois en Top 14, un en Pro D2 ; quatre superviseurs différents ont contribué à ce travail. 
Quelques jours avant chaque rencontre, nous avons contacté le superviseur par téléphone afin de lui présenter le projet de recherche ainsi que les modalités d'investigation comprenant des temps d'observation et d'entretien. Nous l'avons ainsi sollicité pour un premier entretien avant le match, la possibilité de le suivre et de l'observer tout au long de la rencontre puis d'effectuer avec lui, juste après le match puis après la rédaction de son rapport, deux autres entretiens (voir ci-après). Nous avons aussi négocié avec lui les traces écrites, audio, vidéo qu'il était possible de construire et auxquelles il était possible d'avoir accès. Cette négociation s'est faite au cas par cas en fonction du contexte général de la rencontre et de l'appréciation qu'en avait le superviseur. Nous avons enfin indiqué à chaque superviseur que tous les matériaux issus des observations et/ou des entretiens étaient strictement confidentiels, traités de manière anonyme et sans isoler les matches.

Le jour du match, la première rencontre entre le chercheur et le superviseur a lieu avant l'entrée dans le stade. Avant le match, un temps d'entretien (plus ou moins formel) nous permet d'interviewer le superviseur sur la manière dont il s'y prend en match pour réaliser son évaluation de la prestation de l'arbitre. A cette occasion, il nous présente les outils utilisés pendant le match - grille d'observation officielle ou adaptée, dictaphone... - et les différents temps de son activité. Puis, durant tout le temps d'avant match, comme pendant la rencontre, nous accompagnons le superviseur dans les couloirs du stade, dans les vestiaires, dans les tribunes. Au cours de cette phase, notamment pendant le match en tribunes, à chaque fois que cela est possible, nous demandons au superviseur l'autorisation d'enregistrer avec un dictaphone l'ensemble de ses propos et de nos échanges. À la fin du match, nous sollicitons le superviseur sur ses impressions, son appréciation de la prestation de l'arbitre et du contexte de la rencontre. Nous le suivons ensuite jusqu'à la sortie du stade. Durant cette dernière phase, nous tentons d'amener le superviseur à nous indiquer le travail qu'il va effectuer ensuite : les modalités selon lesquelles il pense re-visionner le match à partir de supports vidéo fournis par les diffuseurs, la temporalité qu'il prévoit dans le travail d'écriture de son rapport...

À la suite de chaque rencontre, nous avons construit un journal de bord détaillé rendant compte de l'activité du superviseur et de notre interaction avec lui tout au long de l'investigation. Le superviseur de son côté nous a transmis une photocopie de l'ensemble des documents qu'il a produit pendant et après le match : fiche d'observation, prise de notes spontanée pendant le match et le rapport transmis à la fédération. Ensuite, nous sollicitons un ultime entretien téléphonique avec le superviseur. Ce dernier a eu lieu dans un délai de 15 jours, si possible sans nouvelle supervision intercalée. Cet entretien se structure en plusieurs temps. Il s'agit en premier lieu de revenir sur l'évaluation de la prestation de l'arbitre lors du match. Ainsi, nous nous sommes entretenus avec le superviseur sur notre interprétation de son activité en tribune : ses appréciations, ses éventuels questionnements en situation et la manière dont il construit des traces. Ceci nous a permis de compléter notre compréhension de ce qu'il effectue en match. Puis, nous avons sollicité le superviseur pour qu'il décrive le travail effectué depuis le match: le type d'utilisation de la vidéo, la manière dont ses premières appréciations ont ou non été modifiées par ce travail et les modalités d'écriture de son rapport. Puis dans un second temps, l'entretien vise à spécifier tant le parcours du superviseur - en tant qu'arbitre puis en tant que cadre de la fédération - que sa représentation du «bon » arbitrage et d'une « bonne » supervision.

\section{2.- La construction progressive, par les superviseurs, de la performance arbitrale}

Le travail mené montre la diversité et la richesse des pratiques des superviseurs, mais surtout la complexité de l'établissement de la performance arbitrale.

Le rapport caractérise in fine la prestation de l'arbitre au cours d'un match. La figure 1 est un exemple de rapport de supervision rendu à la fédération. 

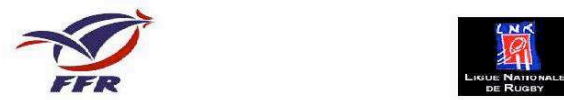

RAPPORT DE SUPERVISION DES OFFICIELS DU MATCH

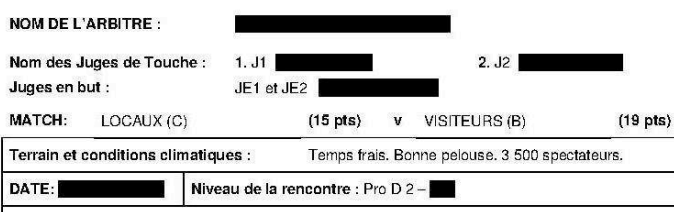

\begin{tabular}{l|l}
\hline DATE: & Niveau de la rencontre : Pro D 2 \\
\hline DESCRIPTION DU JEU:
\end{tabular}

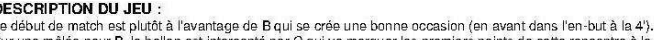

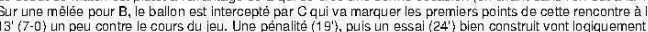

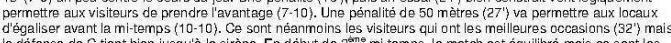

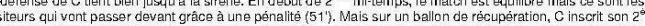

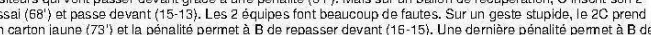

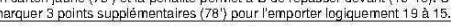
PRESTATION GLOBALE DE L'ARBITRE (et des juges de touche)

Clements de performance positifs:

Borne phoiographie de la phass olacueur-plaqué, viglant sur les entrées illicites.

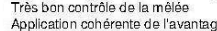

: Borne photograghie des hors jeu dans le jeu couran

Eléments de la parformance nécessitant une amelioration

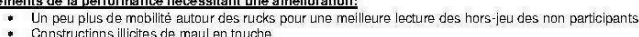
PERFORMANCE D'ENSEMBLE:

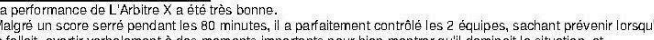

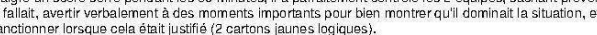
idé par la bonne discipline des 2 équipes, sa première ni temps a été remarcuable evec très peu de déchets

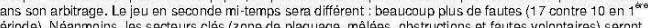

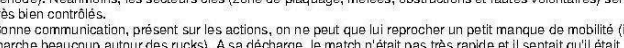
narche beaucou
oujou's présent.

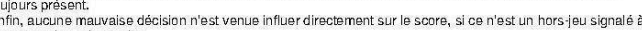

TRESTATION DES JUGES DE TOUCHE ET Cl I'ARBITRE VIDEO:

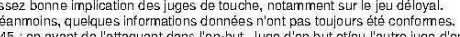

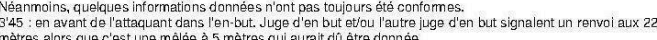

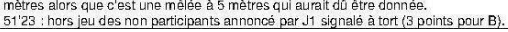

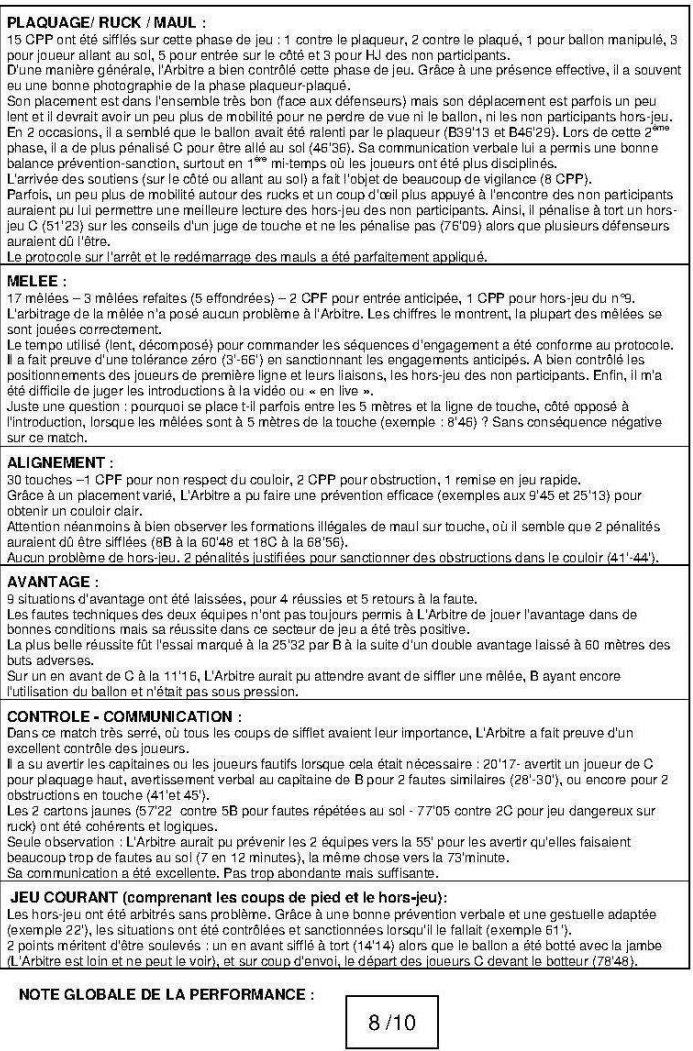

$8 / 10$

Figure 1 : Exemple de rapport de supervision

Figure 1: Example of a supervision report

Le superviseur attribue une note à l'arbitre et doit renseigner plusieurs items pré-déterminés. Les items sont les suivants: Description du jeu, Prestation globale de l'arbitre, Plaquage/Ruck/Maul, Mêlée, Alignement, Avantage, Jeu courant, Contrôle/ Communication. Ainsi, ces items sont relatifs d'une part à la prestation globale de l'arbitre, d'autre part à des phases de jeu particulières et au rapport de l'arbitre aux joueurs. Chaque item est renseigné en pointant d'une part les aspects positifs, d'autre part ceux à améliorer. Si le rapport est le produit final qui constitue en tant que telle l'évaluation de la prestation de l'arbitre, la performance arbitrale se construit tout au long de la supervision. Nous avons considéré les modalités de construction de la performance arbitrale afin de mieux la caractériser. Nous avons pu identifier plusieurs processus contribuant à cette construction .

Il faut tout d'abord souligner que les superviseurs pendant le match produisent une description fine, largement factuelle, de la succession des événements du match. Cette description est produite à partir de grilles d'observation proposées par la fédération ou à l'aide d'outils que le superviseur s'est lui-même construit - grille simplifiée, description verbale enregistrée par dictaphone... Quelles que soient les techniques utilisées, les superviseurs s'attachent à décrire finement au fur et à mesure de la rencontre l'avènement et l'issue de certaines phases de jeu - touche, mêlée, plaquage... - qu'elles fassent ou non l'objet d'une intervention de l'arbitre. Ainsi, les grilles officielles ne sont pas des grilles d'évaluation, puisqu'elles ne contribuent pas à donner une valeur, mais des grilles d'observation qui servent à construire une trace du déroulement du match. Ce relevé factuel peut éventuellement être complété lors du travail à partir de la vidéo du match.

Si la production d'une description fine du déroulement du match est un processus important dans l'évaluation de la prestation de l'arbitre, ce qui est alors produit n'a pas de caractère évaluatif. Ceci ne signifie pas que le superviseur n'attribue aucune valeur à l'arbitrage 
pendant le match, bien au contraire. Mais, il distingue son relevé factuel des jugements évaluatifs qu'il émet. En effet, il produit une autre trace, écrite ou verbale, largement spontanée qui cristallise ses jugements évaluatifs ponctuels, ciblés sur des moments précis. En dehors de la grille d'observation, un superviseur inscrit par exemple sur une feuille volante divisée en deux colonnes ses jugements évaluatifs ponctuels positifs d'un côté, négatifs de l'autre. Il attribue alors une valeur positive ou négative à ce qu'il observe. Nous avons pu relever que ces jugements évaluatifs peuvent porter sur :

1. l'activité des joueurs; par exemple le fait qu'ils soient ou non disciplinés ou qu'ils aient ou non une belle attitude au sol,

2. l'activité de l'arbitre pour elle-même : sa gestuelle lors du signalement d'une faute ou la manière de s'adresser à un joueur,

3. l'activité de l'arbitre comme directement intégrée au jeu ; le déroulement du jeu est alors envisagé comme le fruit d'une co-construction entre les joueurs et l'arbitre; les conséquences directes de l'activité de l'arbitre sur celles des joueurs sont dans ce cas particulièrement scrutées.

Le superviseur produit ainsi, parallèlement à sa description du déroulement du jeu, des jugements évaluatifs concernant des actions ponctuelles, circonscrites dans l'espace et le temps. Mais la performance arbitrale ne se réduit pas à une somme de réussites ou d'échecs par rapport à des épisodes particuliers. En effet, les superviseurs construisent des liens logiques entre les jugements évaluatifs locaux pour établir la performance arbitrale dans des jugements de pratique globaux. C'est relativement à la continuité ou aux fluctuations des jugements évaluatifs locaux - positifs ou négatifs - liés à des types de situations, que se construisent des jugements de pratique globaux. Ces derniers instituent alors des situations comme exemplaires de performance ou de contre-performance et/ou établissent les causes des fluctuations. La performance arbitrale pour un match donné est donc bien construite progressivement dans une enquête qui établit des épisodes comme exemplaires de la prestation de l'arbitre et projette des explications concernant les perturbations et les variations. Ainsi, nous soulignons que non seulement la performance arbitrale ne se réduit pas au cumul de jugements évaluatifs circonscrits à des phases de jeu isolées, mais aussi qu'elle se construit dans une historicité. Cette historicité est d'autant plus constitutive de la performance que les jugements évaluatifs du superviseur s'inscrivent dans une filiation et s'enchâssent les uns dans les autres. En effet, même si la performance arbitrale se construit tout au long du match, les premiers jugements évaluatifs locaux du superviseur dans le match sont cruciaux dans la mesure où ils semblent orienter les suivants. Les premiers jugements évaluatifs locaux amorcent des focalisations particulières et orientent le travail d'enquête constitutif de la supervision. Par exemple, si lors du signalement des premières fautes le superviseur attribue une valeur négative à la gestuelle de l'arbitre, celle-ci va faire l'objet d'une focalisation particulière dans la poursuite du match. Il ne s'agit donc pas d'une impression que le superviseur va chercher à conforter, mais d'une focalisation qui se construit en fonction de ses premiers jugements évaluatifs locaux. Ainsi, l'évaluation ne se réduit pas à une appréciation selon des critères totalement prédéterminés, mais les éléments appréciés lors de la supervision sont contextualisés au sens où ils se construisent au fil des jugements évaluatifs locaux qui orientent la(les) focalisation(s) future(s) du superviseur.

Pour comprendre les modalités de construction de la performance arbitrale, nous avons également tenté de circonscrire ce qui fonde l'attribution de valeurs positives ou négatives au cours d'un jugement évaluatif. Selon Dewey (Dewey, 1939 ; Dewey \& Bidet, 2008), ce sont les intérêts qui fondent l'attribution de valeurs au cours d'un «processus de valuation »; processus de valuation au cours duquel s'originent les jugements évaluatifs. Nous avons donc considéré les intérêts du superviseur, intérêts qui organisent son activité perceptive et évaluative, afin d'approcher ce qui est central dans la construction de la performance arbitrale. Tout au long de leur expérience dans l'arbitrage, les superviseurs construisent et 
intériorisent des intérêts spécifiques relatifs au jeu et à l'activité arbitrale. Ces intérêts, fruits d'une culture sportive et arbitrale évolutive, orientent le «processus de valuation» vers certains objets particuliers et leurs relations. Ces intérêts définissent en partie ce à quoi s'attache le superviseur pour établir une performance arbitrale : ce ne sont ni des critères, ni des éléments objectivés, mais plutôt des centrations qui fondent l'attribution spontanée de valeurs à la prestation de l'arbitre. Nous avons pu identifier plusieurs intérêts communs aux superviseurs dont l'activité a été étudiée :

1. les protocoles utilisés par l'arbitre : les superviseurs se saisissent ainsi de la manière dont l'arbitre donne ses commandements en mêlée, dont il indique aux joueurs les différents temps du maul ou dont il montre qu'une touche est terminée ;

2. le rapport de force dans le jeu : les superviseurs attribuent spontanément une valeur au rapport de force dans le jeu, à l'appréhension qu'en a l'arbitre et à sa gestion de ce rapport de force; ils construisent pour ce faire différents indicateurs, par exemple le nombre de turn-over - ballon perdu au sol - ou le nombre de fautes concédées ;

3. les principes éthiques : les superviseurs appréhendent spontanément «ce qui se fait» ou « ne se fait pas» sur un terrain de rugby tant en termes de pratiques, certaines étant d'emblée déloyales et/ou dangereuses qu'en termes de communication entre l'arbitre et les joueurs relativement à leurs statuts respectifs.

Ces intérêts nous renseignent sur ce qui importe dans la performance arbitrale, mais ils ne définissent pas un bon arbitrage. En effet, la performance arbitrale est construite en contextualisant l'attribution spontanée de valeurs, c'est-à-dire en rapportant cette dernière à des éléments objectivables du contexte. Les jugements évaluatifs du superviseur se fondent tant sur ce processus de valuation où s'origine l'attribution de valeurs que sur la mobilisation d'éléments factuels qu'il relève dans sa description fine du déroulement du match. Ainsi, la performance arbitrale se construit dans un travail d'enquête mettant à l'épreuve d'éléments objectivés, une perception spontanée et subjective. La performance arbitrale ne peut donc être rapportée ni à quelques éléments objectifs qui pourraient faire l'objet d'un traitement statistique, ni à une appréhension totalement subjective. Elle se construit dans un travail d'objectivation : l'attribution de valeurs première est confrontée à des éléments objectivés inscrits dans une description du match qui permettent soit de la fonder, de l'établir, soit de la remettre en cause. Les jugements évaluatifs locaux et globaux constitutifs de la construction de la performance arbitrale s'élaborent à l'interface de valeurs subjectives - plus ou moins partagées au sein d'une même communauté - et de la recherche d'une objectivité.

\section{3.- La performance arbitrale : complexité et inscription systémique}

Au terme de cette étude de l'activité des superviseurs, nous pouvons souligner que la performance arbitrale n'est pas relative à une somme de réussites ou d'échecs par rapport à des épisodes particuliers. Par contre, certains épisodes sont mobilisés dans des jugements globaux en ce qu'ils sont exemplaires de la prestation de l'arbitre. Une part de la complexité de la performance arbitrale semble donc résider dans le fait qu'elle se construit dans un jugement synthétique qui prend en compte la dynamique de la prestation de l'arbitre tout en mobilisant des épisodes ponctuels. La complexité de la performance arbitrale paraît aussi relative à sa construction à l'interface des intérêts des superviseurs - des centrations propres à une communauté d'experts - et d'éléments objectivés plus facilement appréhendables par tous. Enfin, elle n'est pas rapportable à des critères totalement prédéterminés. Les éléments appréciés sont contextualisés, au sens où ils se construisent au fil de l'évaluation, par le superviseur, de la prestation de l'arbitre.

Si ce travail caractérise la manière dont la performance arbitrale est établie par l'institution, il nous permet aussi d'appréhender les attentes qui pèsent sur l'activité de l'arbitre sur le terrain. En effet, la complexité de la performance arbitrale peut engendrer pour l'arbitre une difficulté de lisibilité de l'évaluation qui peut être faite de sa prestation. Même si un 
jugement synthétique prenant en charge la dynamique de la prestation est premier, il reste que certains épisodes d'arbitrage peuvent devenir cruciaux d'une part en ce qu'ils orientent la focalisation du superviseur en fonction de la valeur attribuée à ce moment-là, d'autre part en ce qu'ils peuvent être instaurés comme exemplaires d'une prestation. De même, la construction progressive des éléments appréciés, comme les supports à la fois objectifs et subjectifs du travail d'évaluation, bien que nécessaires, peuvent rendre les attentes, qui pèsent sur l'arbitre, variables et peu anticipables pour lui. De plus, même si les superviseurs partagent un certain nombre d'intérêts - au sens de centrations -, certains sont aussi propres à chacun et à leurs inscriptions particulières dans le monde du rugby et de l'arbitrage en particulier, à leurs statuts et aux responsabilités qu'ils exercent au sein de l'arbitrage français et international. En effet, les intérêts se construisent et se reconstruisent dans un couplage entre l'individu et son environnement; par conséquent plus les contextes dans lesquels ils évoluent sont différents plus leurs intérêts peuvent l'être. Cette variabilité des intérêts des superviseurs peut constituer un frein à la prestation de l'arbitre et diminuer le crédit que les arbitres accordent à la supervision. À cela il faut ajouter que l'arbitre et le superviseur ne sont que deux des acteurs de la performance arbitrale. Les joueurs, les entraîneurs, la direction technique nationale de l'arbitrage au sein des fédérations, la ligue, la fédération, voir les médias... sont autant d'autres acteurs dont l'activité conditionne également la performance arbitrale de différentes manières et à des degrés divers. Chacun des acteurs juge donc la prestation de l'arbitre et contribue à établir la performance arbitrale à l'aune de ses critères et de ses intérêts. Par exemple, ce qui semble important aux yeux des joueurs et des entraîneurs, c'est d'une part, la cohérence des décisions d'un arbitre au fil d'un match, d'un week-end à l'autre, d'un arbitre à l'autre, et d'autre part le fait qu'il soit garant d'une égalité dans la confrontation sans favoriser l'une ou l'autre des parties. Les médias s'attachent essentiellement à la valeur de la prestation de l'arbitre lors d'épisodes ponctuels et rapportent cette dernière aux éléments que les outils vidéo factualisent. Ainsi différentes tensions sont au cour de la construction de la performance arbitrale, tension entre :

1. réussites ou échecs relatifs à des épisodes particuliers et appréciation globale de la dynamique de la prestation dans un jugement synthétique : par exemple, un entraineur va remettre en cause la légitimité d'un carton attribué à un joueur à un moment donné au regard de la faute sifflée alors qu'un superviseur va valoriser le contrôle au fil du match et la gestion générale de la rencontre auxquels les attributions de cartons ont contribué ;

2. les éléments objectivés et les intérêts propres à chaque communauté : un en-avant va être considéré par un commentateur TV en fonction d'un ralenti qui lui permet d'établir si les mains du passeur et/ou la trajectoire du ballon partent vers l'avant, alors que l'entraineur du joueur en question mettra en exergue que sur une passe de la main à la main - très courte - l'en-avant est négligeable et que ce type de passe n'a jamais été sifflée comme un en-avant ;

3. les critères posés a priori et dans l'absolu et les éléments d'appréciation construits en situation: les sollicitations, par l'arbitre, de l'arbitre vidéo sont encadrées par des protocoles précisant les moments où il peut être sollicité et les informations qu'il peut transmettre; dans certains cas, l'arbitre est pris entre le respect de ce protocole et le besoin de confirmation de certaines informations - qui peuvent être hors protocole - pour fonder son jugement et mieux contrôler le match ;

4. une prestation individuelle de l'arbitre et une performance co-construite avec les joueurs : faire refaire à plusieurs reprises une même mêlée signifie pour certains observateurs, que l'arbitre « ne sait pas ce qu'il se passe dans la cage » alors que pour l'arbitre et d'autres observateurs, le temps qu'il prend pour imposer des temps d'entrée et/ou des postures aux joueurs est important pour établir des modalités de coordination performantes.

Les tensions repérées ici ne sont pas exhaustives, mais elles suffisent à souligner la complexité de l'activité d'arbitrage, de la construction de la performance arbitrale et son caractère systémique. Ceci renforce la nécessité de se détacher d'une représentation de la 
performance arbitrale comme étant celle d'un individu unique ; nous montrons comment elle s'inscrit dans un système.

Comme certaines études sur les métiers dans le domaine des services le montrent (Caroly, \& Weill-Fassina, 2004 ; Motté, \& Haradji, 2007), l'opérateur central - ici l'arbitre, mais comme le vendeur en ligne ou le guichetier - est tiraillé entre la satisfaction des attentes de l'institution, celle des attentes des co-acteurs de la prestation et ce qu'il considère comme devant être fait. Dans le cas de l'arbitrage, les attentes des observateurs - médias et spectateurs - doivent aussi être considérées ; elles pèsent sur la prestation de l'arbitre. En effet, cette dernière est publique tout comme le produit qu'elle contribue à construire, le jeu. Mais ce que doit être le jeu, ce bien public, se pose d'autant plus que chacun se l'approprie et en a une appréhension particulière - tout comme le bon travail. La difficulté extrême de l'arbitrage, et en cela il constitue un cas exemplaire, c'est justement d'avoir à faire face en direct - au cours même de son activité -, comme en différé, aux diverses appréciations de sa prestation et indissociablement du jeu produit. La diversité de ces attentes engendre des contradictions que l'arbitre, comme opérateur, doit gérer en situation. Dans d'autres métiers, il est reconnu que ces tensions, ces contradictions, peuvent être à l'origine de problèmes de santé au travail et de détérioration de la performance (Caroly, \& Weill-Fassina, 2004). Elles le deviennent si elles ne sont pas travaillées en tant que telles de manière à passer d'un statut d'empêchement à une opportunité de développement (Clot, 1999). Pour devenir une opportunité de développement, ces tensions ne doivent plus être de l'ordre de conflits intrapsychiques, mais supposent d'être mises à jour, explicitées et discutées dans une démarche soutenue par l'organisation. Notre travail qui dévoile des tensions entre des enjeux contradictoires et des différences dans l'appréciation de la performance met ainsi en évidence tant une contrainte pouvant peser sur l'activité d'arbitrage que les possibles marges de manœuvre de l'arbitre pour s'ajuster au plus près aux différents enjeux spécifiques et contradictoires de l'activité. Nous soulignons ainsi l'importance, dans des activités qui comme l'arbitrage laisse une large part à la créativité et l'initiative de l'opérateur d'identifier les tensions sous-jacentes à la construction de la performance afin de mieux définir les marges d'initiatives des acteurs pour favoriser leur créativité (Lièvre, \& Coutarel, 2013). Autrement dit, dans une économie de la connaissance valorisant l'innovation, la mise à jour des tensions sous-jacentes à la performance peut constituer un outil pour reconnaitre et soutenir la créativité. La médiatisation de l'arbitrage sportif et son caractère public exacerbent les tensions sous-jacentes à la performance et les rendent plus facilement visibles ; son étude permet ainsi de révéler les enjeux de la mise à jour des tensions sousjacentes à la performance dans d'autres activités professionnelles plus discrètes, mais traversées par les mêmes problématiques.

\section{3.- Les disputes de métier, une voie de développement de la performance arbitrale}

Les deux études menées mettent en évidence différents déterminants de la performance arbitrale. Elles montrent d'une part que si le potentiel physique, le savoir réglementaire et l'acuité perceptive sont importants, la prestation de l'arbitre repose sur des compétences et des connaissances qui dépassent largement ces exigences. L'arbitre construit différentes modalités d'appréhension de l'activité des joueurs, différentes modalités de coordination avec eux et assume l'obligation de juger dans une situation antagoniste. D'autre part, les connaissances et compétences de l'arbitre ne sont pas les seuls déterminants de la performance arbitrale.

Au-delà d'une connaissance plus approfondie de l'activité effective de l'arbitre et d'une perspective plus systémique sur la performance arbitrale, ces études participent également à l'évolution de l'arbitrage. Il faut tout d'abord souligner que le travail mené avec les arbitres et les superviseurs suscite leur réflexivité, leur permet de mettre en mots une pratique qui en 
manque toujours et contribue ainsi à leurs développements personnels. Mais la question posée est surtout celle des leviers de développement du métier. Même si ces travaux n'ont pas été négociés comme une intervention, la collaboration avec la FFR sur le long terme permet d'envisager des actions communes et un accompagnement dans la transformation. Dans ce sens, il est prévu une restitution du travail à l'ensemble des superviseurs du hautniveau ; restitution qui a été pensée par la Direction Technique Nationale des Arbitres, comme le moyen d'instaurer des temps d'échanges et de travail plus importants entre superviseurs. Les résultats de ces études font aussi l'objet de discussions collectives au sein d'une formation organisée par l'université en partenariat avec la FFR, le Diplôme d'Université «Sport de haut-niveau et arbitrage ». À cette occasion, les arbitres de hautniveau discutant les résultats construisent d'autres manières d'envisager l'arbitrage et d'en rendre compte. On voit ainsi leurs discours dans la presse se transformer en positionnant par exemple ce que peut-être une vidéo, quand bien même serait-elle une Ref Cam - caméra placée par Canal + sur certains arbitres pendant des matches de Top 14 pour offrir au grand public une autre perspective -, par rapport à la perception d'un arbitre. Nous avons enfin été invités à participer à différentes actions de valorisation de l'arbitrage auprès du grand public ; l'enjeu étant de porter la conception alternative de l'arbitrage et de la performance arbitrale que nous avons construite.

Mais ces travaux nous conduisent aussi à envisager plus largement une voie de développement de la performance arbitrale en mettant au centre son inscription organisationnelle, notamment en prenant en charge les tensions constitutives de la construction de la performance arbitrale. Si la présence de logiques contradictoires et de critères divergents est le lot de tout système social, de nombreux travaux dans le monde du travail ont pu insister sur le rôle que pouvait jouer l'organisation au sein du système (Guérin, Laville, Daniellou, Duraffourg, \& Kerguelen, 1997). À la suite de Clot et des travaux menés en clinique de l'activité (Clot, 1999; Clot, \& Faïta, 2000), nous envisageons de mettre au centre du développement de la performance arbitrale la mise en débat des tensions constitutives de cette dernière et la confrontation de différentes modalités de gestion de ces tensions. Prises en charge collectivement, la mise en question de la prescription pour la renouveler, la mise en débat de différentes manières de faire, la mise au travail de tensions constitutives de la performance représentent autant de leviers de développement de la performance, mais aussi de construction du métier. Il s'agit de tendre vers la construction progressive d'un genre professionnel, c'est-à-dire vers la définition des " "obligations" que partagent ceux qui travaillent pour arriver à travailler, [... de] ce que les travailleurs d'un milieu donné connaissent et voient, attendent et reconnaissent, apprécient ou redoutent ; [... de] ce qu'ils savent devoir faire grâce à une communauté d'évaluations présupposées » (Clot, \& Faita, 2000, pp.9-11). L'enjeu des «disputes» est à la fois de formaliser temporairement des modalités de définition et d'évaluation de la performance et de faire vivre le métier. Ainsi, cette formalisation doit à la fois établir un cadre - tout n'est pas possible, l'organisation fixe des limites - et permettre le développement de la compétence créatrice (Zarifian, 2009). Le produit du travail collectif, tout en définissant la performance arbitrale, devient alors une ressource pour les arbitres, une possibilité de construire des marges de manœuvre dans l'exercice de leur activité.

Pour que la formalisation de la performance arbitrale soit légitime et devienne une ressource, plusieurs conditions doivent être réunies. En premier lieu, la diversité des «acteurs qui comptent » doit être représentée dans les « disputes » de métier. S'il est aisé d'identifier et de mobiliser certains de ces acteurs - arbitres, superviseurs, direction nationale de l'arbitrage -, un travail doit être effectué pour circonscrire le périmètre des acteurs à mobiliser: quels acteurs du jeu - entraîneurs, joueurs, direction technique nationale -, quels acteurs institutionnels - les dirigeants de la fédération, la commission disciplinaire, les dirigeants de la ligue professionnelle, les dirigeants de clubs -, quels acteurs de l'environnement sportif médias, supporters, spectateurs - ? Des échanges existent déjà entre certains de ces acteurs : 
réunions de rentrée où les arbitres de haut-niveau vont à la rencontre des clubs, invitations d'entraineurs aux stages des arbitres ou encore débats ouverts au grand public « Autour des arbitres » en 2013 pour expliquer les évolutions réglementaires et le rôle de l'arbitre. Mais ces échanges tiennent souvent plus de l'exercice formel que d'un véritable travail collectif. Ils sont importants en ce qu'ils affichent une volonté commune de rapprochement des différents acteurs du sport, cependant ces débats restent au niveau de la tâche plutôt que de s'attacher à la confrontation de leurs activités effectives. Les freins à ce travail collectif, à ce dévoilement des pratiques de chacun, sont nombreux. En effet, le jeu sportif est une situation d'opposition antagoniste - si une équipe gagne, l'autre perd -, une situation compétitive où les ficelles du métier de certains entraineurs ou joueurs, faisant défaut aux autres, peuvent être un facteur concurrentiel, une situation de contre-communication où il est important de masquer aux autres ses intentions et ses atouts pour gagner. C'est donc un contexte où la question du développement conjoint ne va pas de soi. Pourtant, il ne paraît pas impossible. La FFR a déjà mis en place les conditions d'un travail collectif sur la mêlée. Confrontée au coût social, humain et économique que représentaient les blessures entrainant des paralysies lourdes, la fédération a mis en place une « Académie des premières lignes » rassemblant des joueurs experts, des entraineurs, les arbitres professionnels, des dirigeants, des formateurs. Ces différents acteurs ont été réunis à plusieurs reprises au cours d'un travail inscrit dans la durée (environ 4 ans) pour analyser, discuter, repenser les conditions pour une mêlée sécurisée et performante en partant notamment de l'expérience des différents acteurs, mais aussi de résultats scientifiques. Ce travail a permis de repenser la formation des premières lignes et de discuter les règles et les modalités d'arbitrage de cette phase de jeu. Ainsi, un véritable travail collectif pour le développement de la santé et la performance des acteurs est possible même dans le sport de haut-niveau, mais il paraît conditionné en partie à une volonté politique forte: la dynamique permanente de rélaboration des modalités de performance doit ainsi être instituée et soutenue par le système et l'organisation (Baret, \& Robelet, 2010 ; Caroly, 2010 ; Clot, 2010 ; Dugué, \& Petit, 2010 ; Petit, 2005). L'institution doit favoriser la prise en charge par les agents du processus de formalisation et de discussion des tensions constitutives de la performance arbitrale; un processus qui au regard du contexte évolutif du sport professionnel, doit être renouvelé régulièrement. Le projet de construction d'un référentiel d'activité des arbitres de haut-niveau pourrait susciter à la fois l'intérêt des acteurs et obtenir le soutien de l'institution. En effet, la question du statut de l'arbitre est une préoccupation prégnante dans le monde sportif. C'est un sujet qui fait débat ; différentes perspectives s'affrontent. L'engagement des différents acteurs dans un travail sur un référentiel paraît donc envisageable. Ce projet de construction d'un référentiel devrait être en mesure d'amorcer ces «disputes ». Il pourrait même, comme propose Prot (2011), les mettre au centre. En effet, afin de ne pas figer l'activité dans un référentiel - de la garder vivante -, Prot propose d'élaborer ce dernier en partant « de[s] dilemme[s] caractéristique[s] de l'exercice d'un métier» (ibid., p. 199); chacun des dilemmes identifiés au cours du travail de construction du référentiel, ne trouvant pas de résolution simple, mais de multiples voies d'arbitrage construites par les acteurs en situation. Ainsi, une voie de construction du métier et de développement de la performance arbitrale pourrait résider dans la formalisation et la mise en débat des différentes manières d'arbitrer les dilemmes de l'arbitrage.

\section{REMERCIEMENTS}

Nous remercions les experts de la revue qui nous ont permis de préciser et de renforcer à notre propos. Cette recherche ayant été réalisée dans le cadre du PERF Arbitrage (http://perf.arbitrage.univ-bpclermont.fr/), en partenariat avec la Fédération Française de Rugby et avec le soutien du groupe La Poste et de la Région Auvergne, nous remercions également ces institutions pour leur concours. 


\section{BIBLIOGRAPHIE}

Austry, R. (2000). Un déjeuner trop lourd. Rugby Mag, 1001, 45 (écrit en 1993).

Baret, C., \& Robelet, M. (2010). Quelles nouvelles pratiques pour réduire les tensions de la relation patient-soignant à l'hôpital ? In D. Retour, M. Dubois, \& M.-E. Bobillier Chaumon (Eds.), Relations de services. Nouveaux usages, nouveaux usagers (pp. 123-137). Bruxelles: De Boeck.

Caroly, S. (2010). L'activité collective et la réélaboration des règles : des enjeux pour la santé au travail. Habilitation à Diriger les Recherches en Ergonomie, Université Bordeaux 2. Retrieved from http://tel.archives-ouvertes.fr/tel-00464801

Caroly, S., \& Weill-Fassina, A. (2004). Évolutions des régulations de situations critiques au cours de la vie professionnelle dans les relations de service. Le travail humain, 67(4), 305-332.

Clot, Y. (1999). La fonction psychologique du travail. Paris: PUF.

Clot, Y. (2010). Le travail à cœur. Paris: La Découverte.

Clot, Y., \& Faïta, D. (2000). Genre et style en analyse du travail. Travailler, 4, 7-42.

Commission Centrale des Arbitres (2002). Les règles du jeu. Paris: FFR.

Dewey, J. (1939). Theory of valuation. In O. Neurath, R. Carnap \& C. Morris (Eds.), International Encyclopedia of Unified Science $-2^{\text {nd }}$ Volume (pp. 1-67). Chicago: The University of Chicago Press.

Dewey, J., \& Bidet, A. (2008). La théorie de la valuation. Tracés, 15(2), 218-228.

Dosseville, F., \& Garncarzyk, C. (2007). L'arbitrage des pratiques sportives : jugement et décision. Bulletin de psychologie, 60(3), 225-237.

Dugué, B., \& Petit, J. (2010). Concevoir le poste de travail pour concevoir le service, et inversement. In D. Retour, M. Dubois, \& M.-E. Bobillier Chaumon (Eds.), Relations de services. Nouveaux usages, nouveaux usagers (pp. 141-161). Bruxelles: De Boeck.

Favret-Saada, J. (2009). Désorceler. Paris: Éditions de l'Olivier.

Gil, F. (1993). Traité de l'évidence. Grenoble: Millon.

Gilbert, W. D., \& Trudel, P. (2000). A profil of a rule infractions in bantam level Ice Hockey. In A. B. Ashare (Ed.), Safety in Ice Hockey $-3^{\text {rd }}$ volume (pp. 291-301). West Conshohocken, P.A. : American Society for Testing Materials.

Guérin, F., Laville, A., Daniellou, F., Duraffourg, J., \& Kerguelen, A. (1997). Comprendre le travail pour le transformer. Lyon: Éditions de l'ANACT.

Kay, B., \& Gill, N. (2003). Physical demands of elite Rugby League referees: Part one - time and motion analysis. Journal of Science and Medicine in Sport, 6(3), 339-342.

Lièvre, P., \& Coutarel, F. (2013). Sciences de gestion et ergonomie : pour un dialogue dans le cadre d'une économie de la connaissance. Économies et sociétés, 22(1), 123-146.

Mascarenhas, R. D., Button, C., O'Hare, D., \& Dicks, M. (2009). Physical performance and decision making in association football referees: A naturalistic study. Open Sports Sciences, 2(9), 1-9.

Mascarenhas, R. D., Collins, D., Mortimer, P., \& Morris, R. L. (2005). A Naturalistic Approach to Training Accurate and Coherent Decision Making in Rugby Union Referees. The Sport Psychologist, 19(2), 131-147.

Motté, F., \& Haradji, Y. (2007). Le vendeur en ligne dans la relation de service. In M. Zouinar, G. Valléry, \& M.C. Le Port (Eds.), Actes du $42^{\text {ème }}$ congrès de la SELF, Ergonomie des produits et des services (pp. 257-265). Toulouse: Octarès Editions.

Oakeshott, M. (1995). De la conduite humaine. Paris: PUF.

Perelman, C. (1990). Éthique et Droit. Bruxelles: Éditions de l'Université de Bruxelles.

Petit, J. (2005). Organiser la continuité du service : intervention sur l'organisation d'une mutuelle de santé. Thèse de doctorat, Université Bordeaux 2. Retrieved from http://tel.archives-ouvertes.fr/tel00659105

Plessner, H., \& Haar, T. (2006). Sports performance judgments from social cognitive perspective. Psychology of sport and exercise, 7(6), 555-575.

Prot, B. (2011). Apprentissage de la conduite et sécurité routière : un dilemme de référence pour la conception d'un référentiel de diplôme d'enseignant. Activités, 8(2), 189-201. http://www.activites.org/v8n2/v8n2.pdf 
Rainey, D. W., Larsen, J. D., \& Williard, M. J. (1987). A computer simulation of sport officiating behaviour. Journal of Sport Behavior, 10(3), 12-22.

Rains, P. (1984). The production of fairness: officiating in the national hockey league. Sociology of Sport Journal, 1(2), 150-162.

Ricœur, P. (1995). Le juste. Paris: Seuil.

Rix, G. (2005). Typologie des actes de jugement de l'arbitre de rugby expérimenté. Science et Motricité, 56(3), 109-124.

Rix, G., \& Biache, M-J. (2004). Enregistrement en perspective subjective située et entretien en re situ subjectif : une méthodologie de constitution de l'expérience. Intellectica, 38, 363-396.

Rix-Lièvre, G. (2010). Différents modes de confrontation à des traces de sa propre activité. Entre convergences et spécificités. Revue d'anthropologie des connaissances, 4(2), 357-376.

Rix-Lièvre, G., Boyer, S., \& Récopé, M. (2011). Referee cognition as it occurs: different kind of judgment acts. In S. M. Fiore, \& M. Harper-Sciarini (Eds.), Proceedings of the 10th International Conference on Naturalistic Decision Making (pp. 136-139). Orlando: University of Central Florida.

Russel, J.S. (1997). The concept of a call in Baseball. Journal of Philosophy of Sport, 24, 21-37.

Russel, J.S. (1999). Are rules all an Umpire has to work with? Journal of the Philosophy of Sport, 26, 27-49.

Serres, M. (2010). Les arbitres ne font pas d'erreur. In Collectif (Ed.), Tous arbitres (pp. 8-15). Paris: Éditions Chronique.

Theureau J. (1992). Le cours d'action, analyse sémiologique : essais d'une anthropologie cognitive située. Berne: Peter Lang.

Trudel, P., Cote, J., \& Sylvestre, F. (1996). Systematic Observation of Ice Hockey Referees During Games. Journal of Sport Behavior, 19(1), 66-81.

Trudel, P.A., Dionne, J-P., \& Bernard, D. (2000). Differences between assessments of penalties in ice hockey by referees, coaches, players and parents. In A.B. Ashare (Ed.), Safety in ice hockey - 3rd Volume (pp. 274-290). West Conshohocken, P.A.: American Society for Testing Materials.

Valléry, G., \& Leduc, S. (2005). Contribution ergonomique à l'analyse des relations de service. Exemple de professionnalisation d'une fonction d'accueil en bureau de poste. Le Travail Humain, 68(2), 153-189.

Vermersch, P. (1994). L'entretien d'explicitation. Paris: ESF.

Weston, M., Castagna, C., Impellizzeri, F. M., Rampinini, E., \& Abt, G. (2007). Analysis of physical match performance in English Premier League soccer referees with particular reference to first half and player work rates. Journal of Science and Medicine in Sport, 10(6), 390-397.

Zarifian, P. (2009). Le travail et la compétence : entre puissance et contrôle. Paris: PUF.

\section{RESUME}

Cet article prend en charge la question de la performance dans l'arbitrage sportif. Pour la décrire, deux études sont présentées : l'une centrée sur l'activité effective de l'arbitre en partant de son expérience, l'autre sur l'activité du superviseur qui est chargé par la fédération d'évaluer la prestation de l'arbitre. Nous montrons que : (1) la prestation de l'arbitre dépasse la tâche qui lui est généralement assignée : appliquer des règles ; (2) arbitrer suppose de construire une multiplicité de compétences pour montrer et imposer aux joueurs ce qui est possible; (3) la performance arbitrale est co-construite. L'étude de l'activité évaluative des superviseurs complète ces résultats en mettant en évidence les tensions constitutives de la construction de la performance arbitrale : (1) tension entre une performance construite au regard de l'arbitrage d'épisodes ponctuels et une prise en compte de la dynamique de l'arbitrage tout au long du match; (2) tension entre une performance établie relativement à des éléments objectifs et 
une attribution de valeur qui dépend des intérêts d'une communauté ; (3) tension entre des critères posés a priori et des éléments d'appréciation contextualisés. Ces travaux pointent la complexité, le caractère collectif et systémique de la performance arbitrale. Ce faisant, ils nous conduisent à suggérer de nouvelles perspectives de développement de la performance arbitrale fondées sur des « disputes » de métier.

\section{MOTS-CLES}

arbitrage sportif, expérience, compétences, performance, évaluation, disputes de métier

\section{REFERENCEMENT}

Rix-Lièvre, G., Boyer, S., Coutarel, F., \& Lièvre, P. (2014). La performance arbitrale : de son étude à son développement. Activités, 11(1), 86-104, http://www .activites.org/v11n1/v11n1.pdf

Article soumis le 28 avril 2013, accepté pour publication le 2 novembre 2013. 